\title{
A CASE REPORT OF ACINIC CELL CARCINOIMA OF PARAPHARYNGEAL SPACE
}

KEY WORDS:

\section{Dr.}

Padmanabhan

Prabhakar

\section{Dr. Sindhu K*}

\section{Prof R. Kannan}

Assistant Professor , Institute of General Surgery, Madras Medical college and Rajiv Gandhi Government General Hospital, Chennai.

Postgraduate, Institute of General Surgery, Madras Medical college and Rajiv Gandhi Government General Hospital, Chennai. *Corresponding Author

Director , Institute of General Surgery, Madras Medical College and Rajiv Gandhi Government General Hospital, Chennai.

Introduction: Parapharyngeal space is an inverted pyramid shaped space. Bounded superiorly by skull base, inferiorly by hyoid bone, prevertebral fascia posteriorly, buccopharyngeal fascia medially, medial pterygoid, mandibular ramus , parotid laterally . Parapharyngeal tumours are rare and account for only $0.5 \%$ of head and neck tumour. Acinic cell carcinoma of parapharyngeal space is a very rare presentation.

Case Report:

21 year old female with complaint of swelling in left side of neck for lyears 6 month duration, which progressively increasing in size, painless, without any pressure effects.Patient doesn't have any symptoms suggestive of malignancy or tuberculosis.on examination swelling of $7 \times 5 \mathrm{~cm}$ in left side of the neck involving the upper part of anterior triangle. MRI revealed that the swelling could possibly be a vagal Schwannoma. Excision was done. Histopathological examination revealed it to be an Acinic cell carcinoma.

\section{CONCLUSION}

Acinic cell carcinoma of parapharyngeal space is a rare tumour .Parapharyngeal tumours are very difficult to access and require high level of anatomical and surgical knowledge for the treatment.

\section{Acinic cell carcinoma of Parapharyngeal space}

21 year old female with complaint of swelling in left side of neck for lyears 6 month duration, which progressively increasing in size, painless, without any pressure effects. Patient doesn't have any symptoms suggestive of malignancy or tuberculosis On examination ,Single oval shaped swelling in the left lateral aspect of neck, in the upper part of leftanterior triangle measuring $7 \times 5 \mathrm{~cm}$, not warm, not tender, borders well defined, Surface irregular, skin over the swelling pinchable, no scar, no sinus, no distended veins, Variable consistency with area of solid and cystic consistency, mobile in horizontal axis, mobility restricted in vertical axis.Swelling is deep to deep fascia. No fixity to the SCM muscle. No other palpable cervical lymphadenopathy.

Examination of skin of scalp and face, oral cavity were normal. Trachea , Larynx, thyroid gland ,salivary glands were normal on examination. Examination of breast, axillary and inguinal region were normal.

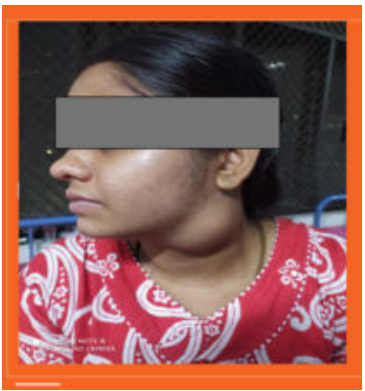

Differential diagnosis that could be given for such swelling will be

- TB cervical lymphadenopathy

- Lymphoma

- Schwannoma

- Paraganglionoma

- Carotid body tumour

- nodal deposits

- Laryngocele

Ultrasound neck revealed a heteroechoic lesion with anterior solid and posterior cystic component. Size $6.5 \times 4.5 \times 5.1 \mathrm{~cm}$ with no increased vascularity, no calcification, involving left level 2 cervical lymphnode and thyroid was normal

MRI neck revealed a heterogenous signal lesion on TIW and T2W images with cystic and solid components in the parapharyngeal and posterior cervical space

Possibility of vagal Schwannoma merits consideration Nodal lesion being a less likely possibility

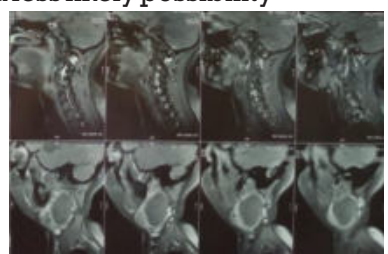

Fnac of the swelling revealed moderately cellular smear with numerous cyst macrophages and few singly scattered polyhedral cells with mucous vacuoles, multinucleated giant cells

\section{Possibility of}

1. Low Grade mucoepidermoid tumor

2. pleomorphic adenoma with cystic changes

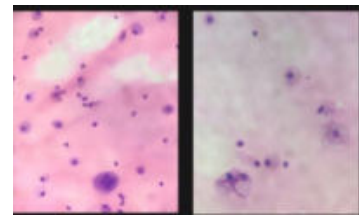

Excision biopsy was planned .Under general anaesthesia Incision made in the lateral aspect of neck extending from mastoid to medial end of clavicle .Intra operative swelling of size $6 \times 5 \times 4 \mathrm{~cm}$ adherent to the carotid sheath.Carotid sheath intact. Swelling removed in toto 


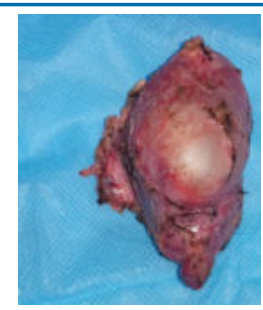

Histopathological examination of the swelling revealed Salivary gland neoplasm- Acinic cell carcinoma

\section{DISCUSSION}

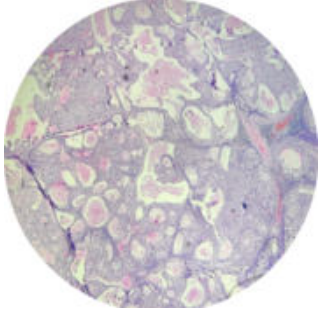

Parapharyngeal space is an inverted pyramid shaped space bounded superiorly by skull base, inferiorly by hyoid bone, posteriorly by prevertebral fascia , medially by buccophar yngeal fascia, laterally by medial pterygoid, mandibular Ramos, parotid.

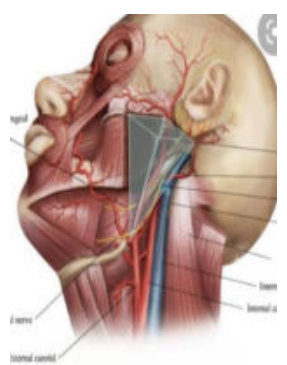

Parapharyngeal space is in turn divided into prestyloid and poststyloid space. Swelling in prestyloid space will be $40-50 \%$ salivary gland neoplasm, $80-90 \%$ will be benign pleomorphic adenoma, $20 \%$ could be malignant, like mucoepidermoid,Acinic cell $\mathrm{Ca}$, carcinoma ex pleomorphic adenoma. Post styloid swelling are mostly neurogenic, in origin. They could be benign like Schwannoma, paraganglioma, neurofibroma, ganglioneuroma or malignant like Malignant paragangliom, Neurofibrosarcoma,Schwannosarcoma, Ganglionoblastoma.

Acinic cell carcinoma is a low or intermediate grade malignant epithelial neoplasm of the salivary glands, characterized by serous acinar cell differentiation. It constitutes $6-8 \%$ of all salivary gland neoplasm. Parotid gland is the most common. Neoplastic cells positive for pan keratin, DOG 1 , SOX10 and PAS D. It shows favorable prognosis, with low rates of local recurrence, distant metastasis and high grade transformation. Wide age range, from children to older adults. Second most common salivary malignancy in children (after mucoepidermoid carcinoma). Third most common salivary malignancy in adults (after mucoepidermoid, adenoid cystic $\mathrm{CA})$. Slightly more common in female than in male( $1.6: 1)$

Most common site is Parotid ( $80 \%)$. Submandibular, sublingual, minor salivary glands rarely involved (13-17\%). Most common site in oral cavity-buccal mucosa and upper lip 